\title{
ANALYSIS OF THE LOGISTICS INTERMEDIARIES CHOICE METHODS IN THE SUPPLY CHAINS
}

\author{
Valery Lukinskiy ${ }^{1}$, Vladislav Lukinskiy ${ }^{2}$ \\ ${ }^{I}$ National Research University Higher School of Economics (HSE) \\ St. Petersburg, Russia \\ Ph.:007(812)644-59-11(+61517), e-mail: lukinskiy@mail.ru \\ ${ }^{2}$ National Research University Higher School of Economics (HSE) \\ St. Petersburg, Russia \\ Ph.:007(812)644-59-11(+61517),e-mail:vladas27@mail.ru
}

\begin{abstract}
The increase of the supply chains efficiency requires optimization of all types of logistic operations and functions. One of such functions is the choice of intermediaries (carriers, freight forwarders, suppliers, service enterprises etc.) and, according to some specialists, it is a major strategic decision in management supply chains. The methods of choosing the logistic intermediaries are considered in many works, however, some questions remain debatable. The article discusses the analytical and expert approaches which serve as a basis to choose the intermediaries; along with it, in the article a comparative evaluation of choice expert methods is done: point-rating assessment, analytic hierarchy process and the general algorithm for selecting; the conclusions have been drawn about the applicability of each method.
\end{abstract}

Keywords: choice of intermediaries, point-rating assessment, analytic hierarchy process (AHP), general algorithm for selecting intermediaries

\section{Introduction}

The increase of the supply chains efficiency requires optimization of all types of logistic operations and functions. One of such functions is the choice of intermediaries (carriers, freight forwarders, suppliers, service enterprises etc.) and, according to some specialists, it is a major strategic decision in management supply chains. It is important to point out that there are some other tasks related to this, for example, infrastructure (warehouse) object choosing, distribution centre location, motion routes, type of transportation, etc.

The logistics development tendencies analysis has shown that in all supply chains sections one can see multi-variance and uncertainty which is expressed in a big amount of intermediaries that can carry out the required conditions and in the presence of the alternative solutions, as well. The logistics intermediaries' choice problem is quite urgent and its correct solution will contribute to the risks decrease and the hardening of the company position at the market.

The article discusses the analytical and expert approaches which serve as the basis to choose the intermediaries; along with it, in the article a comparative evaluation of choice expert methods is done: point-rating assessment, analytic hierarchy process and the general algorithm for selecting; the conclusions have been drawn about the applicability of each method.

\section{Literature review}

We consider it appropriate to draw a quotation of Thomas L. Saaty at the beginning of the review: 'What is the best way of making decision? There are almost half a dozen methods of making decisions which give controversial results for the same data. How can we judge the approach advantages?' (Saaty, 2015, p. 25).

According to (Kopytov and Abramov, 2013), there are various methods that have been developed and implemented to analyse and choose from a range of alternatives using different criteria. The existence of this variety of methods makes the issue of choosing the most suitable one rather difficult.

The issues related to the selection of logistic intermediaries (LI) which are considered almost in all the works on logistics differ mostly by the insight into the matter and the availability of the calculated data. In most works the choice of logistic intermediaries is made under the conditions of certainty and considered to be a single-criterion or multi-criterion problem brought to a single-criterion problem. 
Having analyzed the works of (Bowersox and Closs, 1996; Madera, 2010; Lukinskiy et al., 2012; Saaty, 1994; Taha, 2011 and others) one may distinguish two approaches determining the choice of intermediaries:

- the analytical approach: the choice is based on the formulas with a number of parameters specifying LI (unfortunately, this approach is almost not used in logistics and SCM);

- the expert approach is based on the experts appraisals for the parameters specifying LI and the procedures of obtaining integral expert judgements (ratings).

The expert approach includes at least three main methods:

- $\quad$ point-rating assessment (PRA);

- $\quad$ analytic hierarchy process (AHP);

- general intermediaries choice algorithm (ICA).

The point-rating assessment method (PRA). This approach is based on the attribution of some certain 'weight' to every criterion that reflects its relative importance and alternatives effectiveness evaluation (of the carriers, suppliers, freight forwarders). Rating on every criterion is determined by the multiplication of 'relative importance' and 'effectiveness' evaluations, and the final rating is determined by evaluations addition (Bowersox and Closs, 1996).

The analytic hierarchy process method (AHP) developed by (Saaty, 1994; Saaty 2015) involves consideration of the following hierarchic structure: target - criterion - alternatives.

In the work of (Kabashkin and Lučina, 2015) devoted to the model of decision support for alternative choice, the authors chose the pair wise comparison by the analytical hierarchy process because this method is based on qualitative evaluations. Therefore the results are transferred into a numerical form. The authors believe that the AHP (Analytic Hierarchy Process) is based on paired comparison and seems to be the best choice in this context since it allows structuring the choice procedure as a hierarchy of several levels.

In the work of (Kopytov, Urbach and Labendik, 2013) there is the analysis of the possibility of employing one of the most popular multiple-criteria decision analysis method 'the Analytic Hierarchy Process' to solve the problem of choosing the best method of nano-coatings. According to several authors, AHP method is the most efficient one for the choice of optimal freight transportation system. This provides a wider view of the picture to a decision maker and gives them possibilities for a more flexible process of decision making.

The AHP core lies in defining the relative indexes to evaluate alternative decisions. If we have $n$ criterion or alternatives in the hierarchy background, there must be matrix A sized $n \times n$ created. This matrix is called the pair comparisons matrix and it reflects the decision maker's judgements.

Then, we have to verify the comparison matrix coordination according to the coordination index. In the work of (Taha, 2011) it is mentioned that the comparison matrixes are built according to the human judgements, so it is possible to expect some degree of incoordination and it is necessary to treat it with tolerance provided it stays within some definite 'possible' limitations.

According to (Kopytov and Abramov, 2013), the different groups of criteria have been evaluated by different experts. For instance, the economists have assessed the cost criteria; the transport technologists have evaluated the reliability and ecological criteria, while the managers have estimated the time criteria. The possibility of the pair-wise comparison of a smaller number of criteria in every group allows the experts to determine in a better way the weighted values according to these criteria. The authors have suggested that the number of criteria in each considered group should vary from 3 to 7 . The evaluation of the significance of the criteria groups was determined by the experts with greater qualification.

According to the most experts' opinion, the AHP method enables us to control the experts' judgement solvency and allows increasing the evaluation reliability.

The general intermediaries choice algorithm (ICA) proposed in the work of (Lukinskiy et al., 2012) is, basically, the further development of point-rating assessment method. The ICA is based on the following positions: the criteria are subdivided into three groups: quantitative, qualitative and relay (or 'killer-evaluation'); the simplified pair-comparisons method (or AHP) is used to rank the criteria; quantitative data processing is carried out by the qualimetry methods, and to obtain quality criterion values we suggest to use Harrington desirability function. The calculation of the integral estimates is a sum of qualitative and quantitative criteria evaluation considering the criterion weight.

Unfortunately, among the accessible sources, except for (Lukinskiy and Katkova, 2014), the authors have not managed to find any work containing the comparative evaluation of the depicted methods. So, in spite of the fact that the examined work seems quite simple, in our opinion, there are several aspects of its solution that require additional researches to increase accuracy, transparency and unambiguity of the obtained results. Besides, analysis and systematization of different approaches will allow passing to the development of the combined model which is the synthesis of analytical and expert methods. 


\section{Comparative analysis of the logistics intermediaries choice method}

This methodics approach is intended to clarify and unify the existent methods and intermediaries choice algorithm. And the essence of this approach is to carry out comparative calculations on the same empirical or experimental data. As a result of the analysis and structuring of obtained quantitative evaluations it will be possible to achieve the following objectives:1) to clarify the possibilities of every method; 2) to define the limits of their application; 3) to identify where it is advisable to use various methods altogether (to increase the decisions accuracy and reliability); 4) to classify approaches and methods for solving specific problems of choosing in supply chains in a view of the specifics of the executed logistic functions and operations.

Evidently, along with the relative calculations experience accumulation, there will be an opportunity to define some specific limitations of management decision making on the basis of one or multi-criteria assessments.

Let us examine these indicated intermediaries choice methods in a more detailed way to obtain the goal and to increase the reliability of relative calculations results.

Point-rating assessment method implies the implementation of the following stages.

1. One has to define the alternatives choice criteria and their specific measures $a_{\mathrm{ij}}$ for the goods intermediaries (suppliers) qualities and so on.

2. One has to choose the evaluation principles for each criterion and define their measurements scales.

3. One has to introduce measurement indexes $\mathrm{w}_{\mathrm{i}}$ in the view of each criterion importance.

4. The points calculation is done by the formula

$$
A_{j}=\sum_{i=1}^{n} w_{i} \cdot a_{i j},
$$

where $A_{j}$ is the final point grade of the $j$-th alternative (intermediary); $a_{i j}$ is the indicator (points) for the $i$ th criterion and the $\mathrm{j}$-th alternative.

5. Decision making of the intermediaries choice $A_{\max }$ based on final point grades comparison $A_{j}$ :

$\mathrm{A}_{\min } \leq \mathrm{A}_{1}, \ldots ., \mathrm{A}_{\mathrm{n}-1} \leq \mathrm{A}_{\max }$

The algorithms and integral evaluations calculations examples of logistic intermediaries given in these works differ from each other because the experts participating in the evaluations procedures have not been fully enough formalised and widely varies (for the criteria and alternatives we use points (ranks, weights, ratings) and specific indexes), wherein, their quantity evaluation is often very arbitrary.

On the one hand, this complicates obtaining of final results; on the other hand, this provokes choosing LI in various ways even for the same logistic system because some operations are arbitrary and subjective.

Analytic hierarchy process method. Analysis of the various sources has revealed that AHP covers more and more directions of human activity where it is required to make decision in the conditions of uncertainty or on the basis of the so-called "intangible (psychological, non-material) resources (signs)" (Saaty, 2015).

The calculation order of the weight indexes (criteria and alternatives) matrix according to AHP consists of the following stages.

Stage one. The pair-comparison matrix (PCM) is formed at the each chosen hierarchy level for the criteria and alternatives:

$M=\left(\begin{array}{ccc}1 & a_{12} & a_{13} \\ 1 / a_{12} & 1 & a_{23} \\ 1 / a_{13} & 1 / a_{23} & 1\end{array}\right)$

The experts choose components $\mathrm{a}_{\mathrm{ij}}$ from the so-called fundamental scale: $1,2, \ldots 9$.

The second stage involves receiving the standardised matrix $\mathrm{w}_{\mathrm{ij}}$ (each component is divided into the sum of table components, for example, 1 is divided into $\left(1+1 / \mathrm{a}_{12}+1 / \mathrm{a}_{13}\right)$ and so on: 
$M_{N}=\left(\begin{array}{lll}w_{11} & w_{12} & w_{13} \\ w_{21} & w_{22} & w_{23} \\ w_{31} & w_{32} & w_{33}\end{array}\right)$

Stage three. The average value of the matrix lines components sums give corresponding weight of every criterion or alternative $\mathrm{w}_{\mathrm{n}}$, for example, $\mathrm{w}_{\mathrm{A}}, \mathrm{w}_{\mathrm{B}}, \mathrm{w}_{\mathrm{C}}$. After being ranged the obtained values of the precedence matrix $\mathrm{w}_{\mathrm{n}}$ are the solutions of the problem of resources distribution.

Stage four. At the fourth stage the matrix coordination is checked. To solve this task one has to calculate the proper matrix value $\mathrm{n}_{\max }$ which was received when matrixes $M$ and $\mathrm{w}_{\mathrm{n}}$ had been multiplied. Then, the coordination relation C.R. is calculated by the formula:

C.R. $=\frac{\text { C.I. }}{\text { R.I. }}=\frac{\left(n_{\max }-n\right)}{(n-1) \cdot R . I .}$,

where C.I. is the coordination index of matrix M, and R.I. is the stochastic index of the matrix M coordination.

The values of R.I. for the various order matrixes $n$ are shown in the form of a table (Saaty, 1994) or can be calculated by the formula (Taha, 2011)

$R . I .=\frac{1,98 \cdot(n-2)}{n}$

It is important to emphasise that R.I. is an empiric value which is the average meaning (expected value) of the coefficient C.I. for the large selection of randomly generated inversely proportional matrixes of the $M$ kind.

The size of the 'admissible' limits of C.R. is set in the following way: if C.R. $\leq 0,1$, then the incoordination is acceptable, otherwise, the level is considered to be high and it is recommended to check pair-comparison components to obtain a more coordinated matrix. In the work of (Saaty, 2015) there is the following clarification: for the matrixes of $n=3$ order it is advisable to satisfy the condition C.R. $\leq 0,05$; for $\mathrm{n}=4$ C.R. $\leq 0,08$; and finally, for all the other matrixes we can permit C.R. $\leq 0,2$, but not more than that.

In spite of the increasing popularity of AHP there are still some discussible questions.

1. Why do we need to use the "fundamental scale" of Saaty $(1,2, ., 9)$ for alternatives and criteria having material nature (cost, weight, time etc.)?

2. Is it always necessary to use the fundamental scale for matrixes $3 \times 3,4 \times 4,5 \times 5$ or it is possible to use other scales (for example, Harrington's $(1,2, ., 7)$ ?

3. Why is it thought that the coordination index of $\mathrm{CI}$ is the dispersion of an error the origin of which is conditioned by matrix components evaluation inaccuracy of $a_{\mathrm{ij}}$ ? But what is the source of such an error: expert's opinion or other unknown (inexplicable) reasons?

4. What do we need to do if a matrix appears uncoordinated, i.e. C.R. $>0,1$ ? In this case, according to AHP, it is necessary to study the problem deeper and revise judgments. Attempts to use this recommendation have shown that with seeming simplicity, it is possible to "deform" the matrix of pair comparisons several times and obtain a necessary result, but then the expert practically renounces the authorship.

5. Why can we see the following contradiction: from the 40 matrixes sized of $3 \times 3$ which have been composed by the experts (Saaty, 1994) the coordination C.R. $\leq 0,1$ can be seen in 35 matrixes, i.e. in $88 \%$; in the same work the coordination was $21 \%$ as a result of a statistic modelling of 100 matrixes?

Let us examine the matrix (C.R. $=0,0391)$ to explain this effect:

$M=\left(\begin{array}{ccc}1 & 5 & 3 \\ 1 / 5 & 1 & 1 / 3 \\ 1 / 3 & 3 & 1\end{array}\right)$

Let us do the calculations for the 16 variants and in each of them we will change only one component $a_{13}$, i.e., we will exchange the number 3 into $9,8, \ldots, 1, \ldots, 1 / 9$ (the meaning of $a_{31}$ changes accordingly). In figure 1 there are calculation results that show only when $\mathrm{a}_{13}=1,2$ and 3 we can observe C.R. $\leq 0,1$, that is about $18 \%$. 


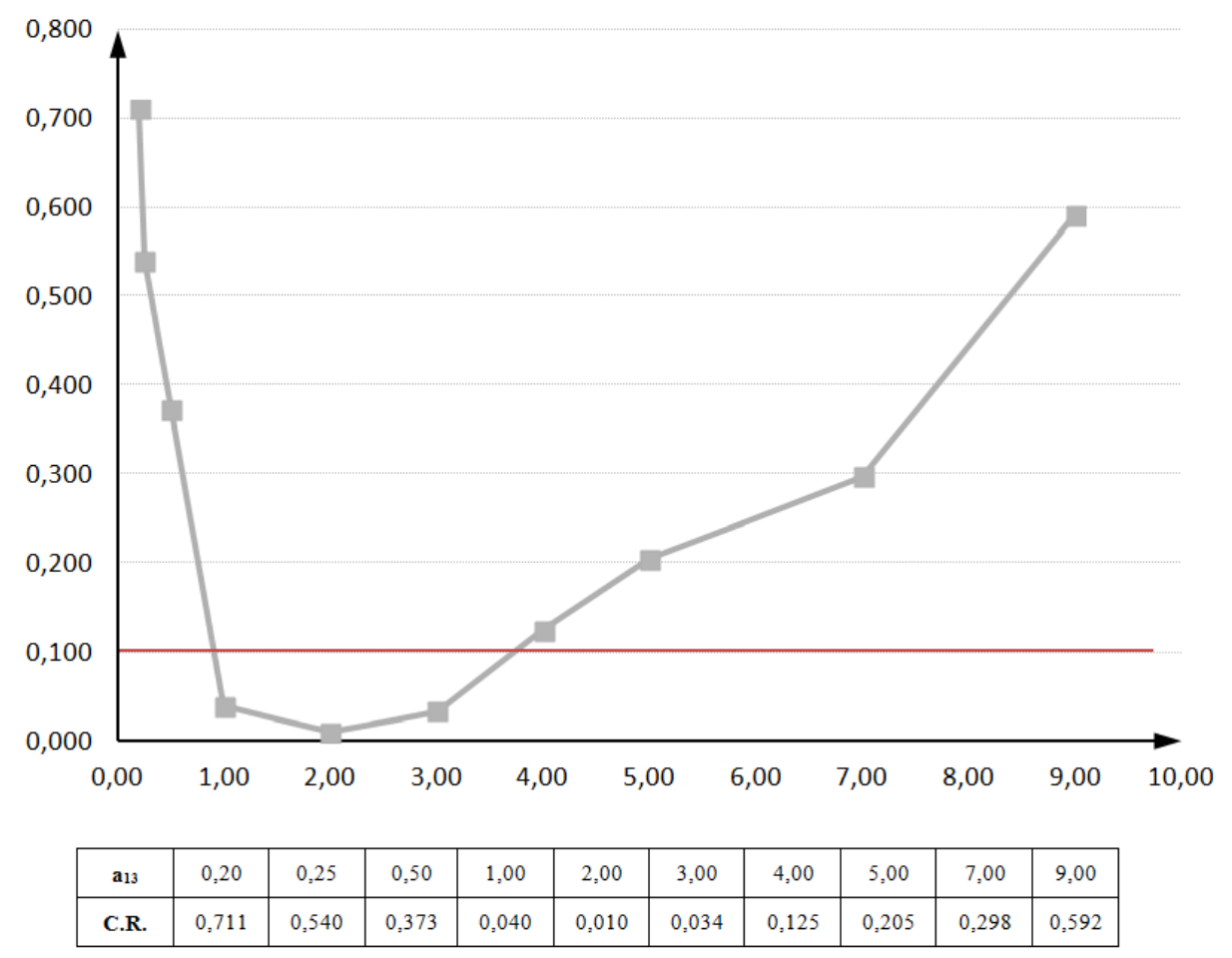

Figure 1. The dependence of C.R. from the component meaning change of PCM

Thus, in spite of the obvious successes of the AHP use in several serious projects, we suppose that some aspects require further research.

The general intermediaries choice algorithm (ICA).

Taking into account the ambiguousness of some positions of AHP, in the work of (Lukinskiy et al., 2012) there was offered an alternative variant of the logistic intermediaries choice in supply chains. The fundamental difference between the ICA and AHP is that ICA provides for the reliable main intermediaries choice while AHP aims at forming the precedence matrix $w_{n}$ which allows distributing of recourses among all the participants (alternatives).

Let us examine the intermediaries choice evaluation order using ICA which algorithm is shown in figure 2. The following modules in this algorithm are of the most interest.

1. The experts rank the indexes using the pair-comparisons; one of the possible variants is to calculate precedence matrix $\mathrm{w}_{\mathrm{n}}$ according to AHP.

2. For the approximation of $\mathrm{w}_{\mathrm{n}}$ the discrete distributions are used, e.g., the one of Poisson's or Fishburn's (Taha, 2011; Fishburn, 1972).

3. The qualimetry methods are used for the intermediaries (alternatives) quantitative indexes; the Harrington's desirability function is used for the qualitative ones.

At the same time the conducted calculations with the use of ICA have shown that some questions need further research, particularly, they need extra variants of applicable distribution laws and the use of the fuzzy sets to evaluate quality indexes, as well.

Summarizing the results of the analysis it is possible to state that each from the examined methods has some certain reliability degree, but it is possible to draw a conclusion about the possible areas of their use only after realization of comparative calculations.

\section{Approbation}

The comparative calculations have been executed for approbation of an offered methodical approach based on the data from the works of (Bowersox and Closs, 1996; Brodetskiy and Terentiev, 2005; Guy and Malakhov, 2011; Madera, 2010; Lukinskiy et al., 2012; Taha 2011). The calculation results are shown in tables $1-5$. Let us examine them in a more detailed way. 


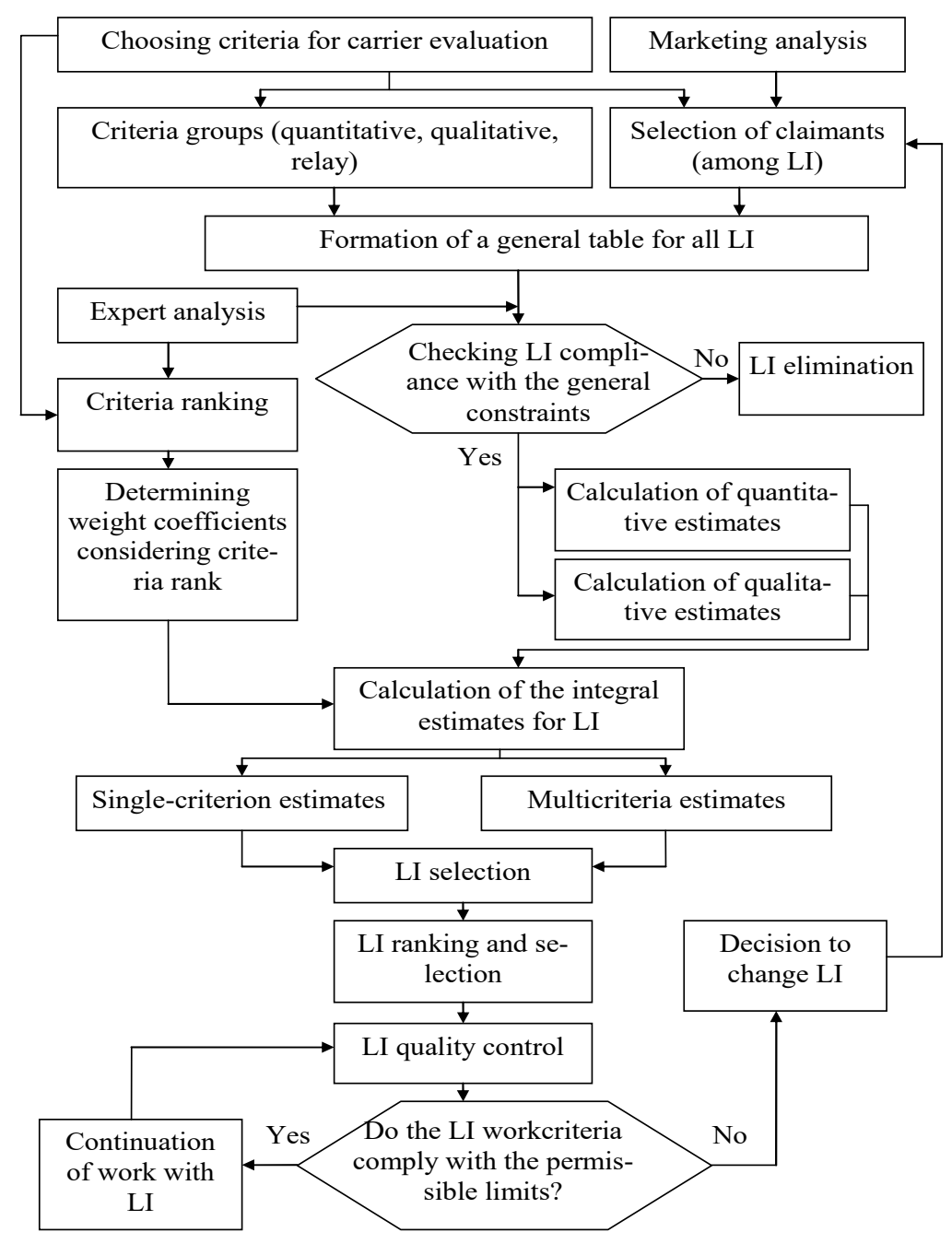

Figure 2. Algorithm for choosing logistic intermediaries

Example 1. In the work of (Lukinskiy et al., 2012) with the help of PRA the carriers choice is done (with 3 alternatives) using 5 criteria (table 1). In this table there are calculations based on ICA, wherein the Fishburn's distribution is chosen for the point indexes evaluation, and the minimum points have been chosen as a standard meaning during the alternative evaluation.

Table 1. Intermediaries choice results comparison

\begin{tabular}{|l|l|l|l|l|}
\hline Method & \multicolumn{1}{|c|}{ Evaluation } & \multicolumn{1}{c|}{ The $\mathbf{1}^{\text {st }}$ carrier } & \multicolumn{1}{c|}{ The 2 The $^{\text {rd }}$ carrier } & \multicolumn{1}{c|}{ carrier } \\
\hline \multirow{2}{*}{ PRA } & Summary rating & 20,99 & 14,94 & 21,02 \\
\cline { 2 - 5 } & Place & $2(3)$ & 1 & $2(3)$ \\
\hline \multirow{2}{*}{ ICA } & Integral evaluation & 0,697 & 0,698 & 0,554 \\
\cline { 2 - 5 } & Place & $2(3)$ & $2(3)$ & 1 \\
\hline \multicolumn{2}{|l|}{ Note: the best evaluation is a minimum rating } \\
\hline
\end{tabular}

Example 2. In the work of (Taha, 2011) the task of choosing the university (3 alternatives) is examined according to two criteria: place and reputation. The corresponding calculations are brought over in table 2, wherein the system analysis and AHP have been used to estimate the values of the criteria and the alternatives.

The calculations of PRA and two variants of ICA have been performed using the same data: in the first one the criteria evaluations were the same as the ones from the work of (Taha, 2011); in the second one the criteria evaluations were calculated by the Fishburn's formula (table 2). 
Table 2. The results comparison of choosing among three universities

\begin{tabular}{|c|c|c|c|c|}
\hline \multirow{2}{*}{ Method } & \multirow{2}{*}{ Evaluation } & \multicolumn{3}{|c|}{ University } \\
\hline & & $\mathbf{A}$ & B & $\mathrm{C}$ \\
\hline \multirow[t]{2}{*}{ PRA } & Summary rating & 31,7 & 18,5 & 18 \\
\hline & Place & 1 & 2 & 3 \\
\hline \multirow[t]{2}{*}{ AHP and system analysis } & Combined weight & 0,4743 & 0,2737 & 0,2520 \\
\hline & Place & 1 & 2 & 3 \\
\hline \multirow[t]{2}{*}{ ICA (the $1^{\text {st }}$ variant) } & Integral evaluation & 0,867 & 0,487 & 0,440 \\
\hline & Place & 1 & 2 & 3 \\
\hline \multirow[t]{2}{*}{ ICA (the $2^{\text {nd }}$ variant) } & Integral evaluation & 0,739 & 0,482 & 0,550 \\
\hline & Place & 1 & 3 & 2 \\
\hline
\end{tabular}

Example 3. In the work of (Brodetskiy and Terentiev, 2005) on the basis of AHP the choice of a city (4 alternatives) is done to optimise the location of the regional distribution centre using 6 criteria. All the values are obtained as a result of the expert survey, that is, without any real data. The calculations results are shown in table 3. In the same table there are ICA calculations results based on the point evaluations of the pair wise indexes and alternatives comparisons, that is, without the matrixes rationing procedure or coordination checking.

Table 3. The comparison results of choosing a location of the regional centre

\begin{tabular}{|c|c|c|c|c|c|}
\hline \multirow{2}{*}{ Method } & \multirow{2}{*}{ Evaluation } & \multicolumn{4}{|c|}{ City (regional centre) } \\
\hline & & $\mathbf{A}$ & B & $\mathbf{C}$ & $\mathbf{D}$ \\
\hline \multirow[t]{2}{*}{ AHP } & Summary rating & 0,458 & 0,309 & 0,129 & 0,112 \\
\hline & Place & 1 & 2 & 3 & 4 \\
\hline \multirow{2}{*}{$\begin{array}{c}\text { ICA } \\
\text { (the } 1^{\text {st }} \text { variant) } \\
\end{array}$} & Integral evaluation & 0,860 & 0,588 & 0,253 & 0,226 \\
\hline & Place & 1 & 2 & 3 & 4 \\
\hline \multirow{2}{*}{$\begin{array}{c}\text { ICA } \\
\text { (the } 2^{\text {nd }} \text { variant) }\end{array}$} & Integral evaluation & 0,827 & 0,619 & 0,291 & 0,265 \\
\hline & Place & 1 & 2 & 3 & 4 \\
\hline
\end{tabular}

In tables 4 and 5 there are the results of the comparative calculations of choosing a location for the supermarket (Madera, 2010) and the terminal (Guy and Malakhov, 2011) correspondingly. The main difference between these calculations and the previous ones is that instead of the points the quantitative and qualitative evaluations have been used for the alternatives. Accordingly, in the same tables there are the ICA.

Table 4. The comparison results of choosing a location of the supermarket construction

\begin{tabular}{|c|c|c|c|c|c|}
\hline \multirow{2}{*}{ Method } & \multicolumn{5}{|c|}{ Construction placement } \\
\cline { 2 - 6 } & A & B & C & D & 2 \\
\hline AHP & 3 & 1 & 5 & E \\
\hline ICA & 2 & 1 & 5 & 3 \\
\hline
\end{tabular}

Table 5. The comparison results of choosing a location of the terminal

\begin{tabular}{|c|c|c|c|c|c|c|}
\hline \multirow{2}{*}{ Method } & \multicolumn{7}{|c|}{ Container terminal location } \\
\cline { 2 - 7 } & A & B & C & D & E & 5 \\
\hline AHP & 1 & 6 & 2 & 4 & 5 & 5 \\
\hline ICA & 1 & 6 & 2 & 3 & 4 \\
\hline
\end{tabular}

\section{Conclusion}

On the basis of the analysis of the executed calculations it is possible to conclude the following.

1. The intermediaries choice results (of suppliers, carriers, locations, etc.) which have been calculated according to the different methods almost coincide, particularly those ones who got the best ratings (places 1 and 2).

2. The ICA method is more difficult that the PRA one, but its objectivity is higher because it allows not only to decrease point assessment variation, but to unite them by the certain patterns, as well. Apart from that, if the alternatives assessments have quantitative (qualitative) evaluations, there is no need for the points use. 
3. We believe that the AHP method possesses substantial advantages without having any quantitative (tangible) information except for the experts' evaluations. At the same time, its laboriousness increases considerably comparing to PRA and ICA (especially within the criteria and alternatives increasing). Besides, it is important to remember about possible difficulties of coordinated precedence matrixes obtaining.

4. It is obvious that the ICA method produces almost the same evaluations as the AHP method does, and can be widely used taking into consideration the simplicity and larger unambiguity, and transparency of the experts' evaluations.

All in all, the development of the combined methodics must be the main direction of the further researches. This methodics consists of two approaches: the first one is analytical, for example, on the basis of total logistics costs (TLC), besides, TLC must include the maximum quantity of factors; the second approach is the expert one. It can include different variants, for example, in the case of an obvious situation it must include PRA; and in the case of moderate uncertainty and risk it must include ICA; under the conditions of total uncertainty it must include AHP.

\section{References}

1. Bowersox, D.J. and Closs, D.J. (1996) Logistical Management. The Integrated Supply Chain Process. New York: McGraw-Hill Companies, Inc.

2. Brodetskiy, G. and Terentiev, P. (2005) Application of an analytical heirarchy method for optimization of a local distribution centre disposition. Logistics and supply chain management. 1(6), 26-34. (In Russian)

3. Fishburn, P.C. (1972) Utility theory for decision making. New York: Wiley.

4. Guy, V. and Malakhov, D. (2011) Choosing a site for an off-dock terminal in the St. Petersburg transport hub using analytic hierarchy process. Logistics and supply chain management. 1(42), 55-59. (In Russian)

5. Kabashkin, I. and Lučina, J. (2015) Development of the model of decision support for alternative choice in the transportation transit system. Transport and Telecommunication, 16 (1), 61-72. DOI: 10.1515/ttj2015-0007.

6. Kopytov, E., Abramov, D. (2013) Multiple-creteria choice of transportation alternatives in freight transport system for different types of cargo. In: Proceedings of the 13th international conference: Reliability and statistics in transportation and communication (RelStat'13). October 16-19, Riga, Latvia, pp. 180-187.

7. Kopytov, E., Urbach, A., Labendik, V. (2013) Comparative multi-criteria assessment of nano-coating technologies. In: Proceedings of the 13th international conference: Reliability and statistics in transportation and communication (RelStat'13). October 16-19, Riga, Latvia, pp. 207-216.

8. Lukinskiy, V.V. and Katkova H. (2014) Analysis of methods for choice of logistics intermediaries. Logistics and supply chain management. 2(61), 49-56. (In Russian)

9. Lukinskiy, V.S., Lukinskiy, V.V., Malevich, J. Plastuniak, I., Pletneva, N. (2012) Models and methods of the logistics theory. St.Petersburg: SPbSUEE.

10. Madera, A.G. (2010) Modelling and decision-making in management. Moscow: LKI. (In Russian)

11. Saaty, T.L. (1994) Fundamentals of Decision Making. Pittsburgh: RWS Publications.

12. Saaty, T.L. (2015) Decision making with dependence and feedback. The Analytic Network Process. Moscow: Lenand. (In Russian)

13. Taha, H. (2011) Operations Research: an Introduction. 9th ed. Upper Saddle River, New Jersey: Prentice Hall. 